Article

\title{
The Bourguignon Laplacian and Harmonic Symmetric Bilinear Forms
}

\author{
Vladimir Rovenski ${ }^{1}(\mathbb{D})$, Sergey Stepanov ${ }^{2, *(D)}$ and Irina Tsyganok ${ }^{3}(\mathbb{D}$ \\ 1 Department of Mathematics, University of Haifa, Haifa 3498838, Israel; vrovenski@univ.haifa.ac.il \\ 2 Department of Mathematics, Russian Institute for Scientific and Technical Information of the Russian \\ Academy of Sciences, Moscow 125190, Russia \\ 3 Department of Data Analysis and Financial Technologies, Finance University, Moscow 125993, Russia; \\ i.i.tsyganok@mail.ru \\ * Correspondence: s.e.stepanov@mail.ru
}

Received: 26 November 2019; Accepted: 24 December 2019; Published: 3 January 2020

check for updates

\begin{abstract}
In this paper, we study the kernel and spectral properties of the Bourguignon Laplacian on a closed Riemannian manifold, which acts on the space of symmetric bilinear forms (considered as one-forms with values in the cotangent bundle of this manifold). We prove that the kernel of this Laplacian is an infinite-dimensional vector space of harmonic symmetric bilinear forms, in particular, such forms on a closed manifold with quasi-negative sectional curvature are zero. We apply these results to the description of surface geometry.
\end{abstract}

Keywords: Riemannian manifold; Bourguignon Laplacian; symmetric bilinear form; harmonic; curvature; spectral theory; vanishing theorem

MSC: 53C20; 53C25; 53C40

\section{Introduction}

In this article, we consider a connected $n$-fimensional smooth manifold $M$ endowed with a Riemannian metric $g$. One can associate a number of natural elliptic differential operators to $(M, g)$, which arise from the Riemannian geometric structure. The most famous one is the Hodge-de Rham Laplacian, $\Delta_{H}$, which acts on $C^{\infty}$-sections of the vector bundle $\Lambda^{p}(M)$ of exterior differential $p$-forms $(p=1, \ldots, n-1)$. For a closed manifold $M$, the condition $\Delta_{H} \omega=0$ defines $\omega$ as a harmonic $p$-form; moreover, the dimension of the kernel $\operatorname{Ker} \Delta_{H}$ is equal to the $p$-th Betti number $b_{p}(M)$ of $M$ (e.g., [1-3] with the fundamentals of the theory of harmonic exterior differential forms on a Riemannian manifold). If the curvature operator $\bar{R}$ of $(M, g)$ is quasi-positive, then $b_{p}(M)=0$. This is one of the main results of the Bochner technique (see ([2], p. 351)), which is used to control the topology of a Riemannian manifold by restrictions on its curvature (see ([2], pp. 333-364)). Many works are devoted to the spectral theory of the Hodge-de Rham Laplacian (e.g., [4]).

J.-P. Bourguignon ([5], p. 273) has defined (as an analogue of $\Delta_{H}$ ) the second order self-adjoint and strongly elliptic differential operator $\Delta_{B}$ acting on $C^{\infty}$-sections of the vector bundle $S^{2} M$ of symmetric bilinear forms on $(M, g)$.

In the paper, we study the kernel and spectral properties of $\Delta_{B}$, considering $S^{2} M$ as one-forms with values in the cotangent bundle of this manifold. We show that on a closed $M$ the kernel Ker $\Delta_{B}$ is a finite-dimensional vector space consisting of harmonic symmetric bilinear forms (see ([5], p. 270) and ([2], p. 350)). Similarly to the Hodge-de Rham Laplacian, $\Delta_{B}$ is also a Laplacian and it admits the Weitzenböck decomposition formula (see [5]). Therefore, we can study the Bourguignon operator using the analytical method, due to Bochner. In particular, we prove that every harmonic symmetric 
bilinear form on a compact $(M, g)$ with quasi-negative sectional curvature is zero. We also investigate the spectral properties of $\Delta_{B}$. In this work, we apply these results to the description of surface geometry. There is not a single article on the spectral theory of the Bourguignon Laplacian, and we are the first to study this theory; thus, our research could have a significant contribution to the general spectral theory of elliptic operators (e.g., [6]). We prove that any harmonic symmetric bilinear form is a Codazzi tensor with constant trace. Therefore, the results in this work can be used in the theory of Codazzi tensors (e.g., ([7], pp. 436-440); [4,8,9]) and its applications in physics (e.g., [10]).

\section{The Bourguignon Laplacian}

Let $(M, g)$ be a closed (compact without boundary) manifold and $L^{2}(M, g)$ be the Hilbert space of functions or tensors with the scalar product

$$
\langle u, w\rangle=\int_{M} g(u, w) \mathrm{d} \operatorname{vol}_{g}
$$

where $\mathrm{d} \mathrm{vol}_{g}$ is the volume form relative to $g$. In this case, $H^{2}(M, g)$ denotes the Hilbert space of functions or tensors determined $(M, g)$ with two covariant derivatives in $L^{2}(M, g)$ and with the usual product and norm.

The cotangent bundle $T^{*} M$ comes equipped with the Levi-Civita covariant derivative $\nabla$; thus, there is an induced exterior differential $d^{\nabla}: C^{\infty}\left(S^{2} M\right) \rightarrow C^{\infty}\left(\Lambda^{2} M \otimes T^{*} M\right)$ on the bundle of $T^{*} M$-valued differential one-forms,

$$
d^{\nabla} \varphi(X, Y, Z):=\left(\nabla_{X} \varphi\right)(Y, Z)-\left(\nabla_{Y} \varphi\right)(X, Z)
$$

for any tangent vector fields $X, Y, Z$ on $M$ and $\varphi \in C^{\infty}\left(S^{2} M\right)$. In particular, if $d^{\nabla} \varphi=0$ then $\varphi \in$ $C^{\infty}\left(S^{2} M\right)$ is said to be a closed bilinear form; in this case, $\varphi$ is a Codazzi tensor. A symmetric bilinear form is called a Codazzi tensor (named after D. Codazzi) if its covariant derivative is a symmetric tensor (see ([7], p. 435); [4,8]). We call a Codazzi tensor trivial if it is a constant multiple of metric. Let $\delta^{\nabla}: C^{\infty}\left(\Lambda^{2} M \otimes T^{*} M\right) \rightarrow C^{\infty}\left(S^{2} M\right)$ be the formal adjoint operator of the exterior differential $d^{\nabla}$ (see ([7], p. 355) and [5]). For an arbitrary local orthonormal frame $\left(e_{i}\right)$ of vector fields on $(M, g)$, we have

$$
\delta^{\nabla} \varphi(X)=-\sum_{i}\left(\nabla_{e_{i}} \varphi\right)\left(e_{i}, X\right) .
$$

Then $\varphi \in C^{\infty}\left(S^{2} M\right)$ is called harmonic if $\omega \in \operatorname{Ker} d^{\nabla} \cap \operatorname{Ker} \delta^{\nabla}$ (see ([5], p. 270) and ([2], p. 350)). Using $d^{\nabla}$ and $\delta^{\nabla}$, J.-P. Bourguignon ([5], p. 273) constructed the Laplacian $\Delta_{B}: C^{\infty}\left(S^{2} M\right) \rightarrow C^{\infty}\left(S^{2} M\right)$ using the formula

$$
\Delta_{B}:=d^{\nabla} \delta^{\nabla}+\delta^{\nabla} d^{\nabla} .
$$

Remark 1. The theory on $T^{*} M$-valued differential one-forms can be found in works from the following list: ([11], p. 338), ([7], pp. 133-134; 355), [5,12], ([2], pp. 349-351).

By direct computations we obtain the following integral formula:

$$
\left\langle\Delta_{B} \varphi, \varphi\right\rangle=\left\langle d^{\nabla} \varphi, d^{\nabla} \varphi\right\rangle+\left\langle\delta^{\nabla} \varphi, \delta^{\nabla} \varphi\right\rangle .
$$

Based on (2), we conclude that the Bourguignon Laplacian $\Delta_{B}$ is a non-negative operator. By the general theorem on elliptic operators (see ([11], p. 383) and ([7], p. 464)), we have the orthogonal decomposition

$$
C^{\infty}\left(S^{2} M\right)=\operatorname{Ker} \Delta_{B} \oplus \operatorname{Im} \Delta_{B},
$$


with respect to the global scalar product $\langle\cdot, \cdot\rangle$. It is known ([7], p. 464) that Ker $\Delta_{B}$ (the kernel of $\Delta_{B}$ ) is a finite-dimensional vector space over the field of real numbers. An easy computation yields the Weitzenböck decomposition formula (e.g., ([7], p. 355), ([5], p. 273)).

$$
\Delta_{B} \varphi=\bar{\Delta} \varphi+B \varphi,
$$

where $\bar{\Delta}=\nabla^{*} \nabla$ is the rough Laplacian (see ([7], p. 52)). The second component of the right hand side of (4) is called the Weitzenböck curvature operator for $\Delta_{B}$. It has the form

$$
B \varphi:=\varphi \circ \operatorname{Ric}-\stackrel{\circ}{R} \varphi,
$$

where $\circ$ is a composition of endomorphisms, Ric is the Ricci curvature, and $\stackrel{\circ}{R}$ is the linear map of $S^{2} M$ into itself such that (see ([7], p. 52))

$$
(\stackrel{\circ}{R} \varphi)(X, Y)=\sum_{i=1}^{n} \varphi\left(R\left(X, e_{i}\right) Y, e_{i}\right)
$$

for the curvature tensor $R$, any $\varphi \in C^{\infty}\left(S^{2} M\right)$ and an arbitrary local orthonormal basis $\left(e_{i}\right)$ of vector fields on $(M, g)$. By the above, $B g=0$ and

$$
\operatorname{trace}_{g}(B \varphi)=0 \text {. }
$$

From (4) and (5) we obtain the identity

$$
\operatorname{trace}_{g}\left(\Delta_{B} \varphi\right)=\bar{\Delta}\left(\operatorname{trace}_{g} \varphi\right) .
$$

We consider the spectral theory of the Laplacian $\Delta_{B}: C^{\infty}\left(S^{2} M\right) \rightarrow C^{\infty}\left(S^{2} M\right)$. Let $\varphi$ be a nonzero eigentensor corresponding to the eigenvalue $\lambda$, that is $\Delta_{B} \varphi=\lambda \varphi$ and $\lambda$ a real nonnegative number. We can rewrite (4) in the following form: $\lambda \varphi=\bar{\Delta} \varphi+B \varphi$. From (6) we obtain

$$
\bar{\Delta}\left(\operatorname{trace}_{g} \varphi\right)=\lambda\left(\operatorname{trace}_{g} \varphi\right),
$$

where $\bar{\Delta}: C^{\infty}(M) \rightarrow C^{\infty}(M)$ is the ordinary Laplacian defined by $\bar{\Delta} f=-\operatorname{div}(\operatorname{grad} f)$ for any $f \in C^{\infty}(M)$. In this case, the following holds:

$$
\left\langle\bar{\Delta}\left(\operatorname{trace}_{g} \varphi\right), \operatorname{trace}_{g} \varphi\right\rangle=\left\langle\nabla \operatorname{trace}_{g} \varphi, \nabla \operatorname{trace}_{g} \varphi\right\rangle .
$$

Therefore (for a closed $M), \bar{\Delta}\left(\operatorname{trace}_{g} \varphi\right)=0$ if and only if trace $\operatorname{s}_{g} \varphi=$ const. We conclude that if (7) holds for trace $g=$ const and $\lambda \neq 0$, then trace $g$ vanishes.

We have proved the following lemma.

Lemma 1. Let $\left(M^{n}, g\right)(n \geq 2)$ be a closed Riemannian manifold and $\Delta_{B} \varphi=\lambda \varphi$ for the Bourguignon Laplacian and for its nonzero eigenvalue $\lambda$. If

$$
\text { trace }_{g} \varphi=\text { const, }
$$

then $\operatorname{trace}_{g} \varphi=0$. On the other hand, if $\operatorname{trace}_{g} \varphi$ is not constant, then $\operatorname{trace}_{g} \varphi$ is an eigenfunction of the rough Laplacian $\bar{\Delta}$ with the same eigenvalue $\lambda$.

\section{Spectrum of the Bourguignon Laplacian}

If the rough Laplacian is defined on scalar functions, then it is called the Laplace-Beltrami Laplacian (see the theory of this Laplacian in [13]). Standard ellipticity theory and the fact that the Laplace-Beltrami Laplacian $\bar{\Delta}$ is a self-adjoint nonnegative elliptic operator imply that the spectrum 
of $\bar{\Delta}$ consists of discrete eigenvalues $0=\bar{\lambda}_{0}<\bar{\lambda}_{1}<\bar{\lambda}_{2}<\ldots$, satisfying the condition $\bar{\Delta} f_{i}=\bar{\lambda}_{i} f_{i}$ for the corresponding complete orthonormal sequence of eigenfunctions $f_{i} \neq 0$ (e.g., [13]). We focus on bounds on the first nonzero eigenvalue $\bar{\lambda}_{1}$ imposed by the Riemannian geometry of $(M, g)$. The lower bound for $\bar{\lambda}_{1}$ was found first by Lichnerowicz ([13], p. 181), and his result is the following: If $\left(M^{n}, g\right)(n \geq 2)$ is a closed Riemannian manifold, whose Ricci curvature satisfies the inequality Ric $\geq(n-1) k$ for some constant $k>0$, then the first positive eigenvalue $\bar{\lambda}$ of the Laplacian $\bar{\Delta}$ has the lower bound $\bar{\lambda} \geq n k$.

Yang [14] generalized the above result in the following form: Let $\left(M^{n}, g\right)(n \geq 2)$ be a closed Riemannian manifold with the diameter $\mathrm{D}(M)$ and condition Ric $\geq(n-1) k$ for some constant $k \geq 0$, then the first positive eigenvalue $\bar{\lambda}$ of the Laplacian $\bar{\Delta}$ satisfies the lower bound inequality

$$
\bar{\lambda} \geq(n-1) k / 4+\pi^{2} / D^{2}(M) .
$$

On the other hand, by the spectral theory (e.g., [13]), $\Delta_{B}$ has a discrete set of eigenvalues $\left\{\lambda_{a}\right\}$ forming a sequence $0=\lambda_{0}<\lambda_{1}<\lambda_{2}<\ldots$, and $\lambda_{a} \rightarrow \infty$ as $a \rightarrow \infty$. Any eigenvalue of $\Delta_{B}$ has finite multiplicity and an arbitrary $\lambda_{a}$ for $a \geq 1$ is positive because $\Delta_{B}$ is a non-negative elliptic operator. As a corollary of the Lichnerowicz and Yang theorems, we obtain the following.

Proposition 1. Let $\left(M^{n}, g\right)(n \geq 2)$ be a closed Riemannian manifold and $\lambda$ a positive eigenvalue of $\Delta_{B}$, such that its corresponding eigentensor $\varphi \in C^{\infty}\left(S^{2} M\right)$ has a nonzero trace. If the Ricci curvature of $(M, g)$ satisfies the inequality Ric $\geq(n-1) k$ for some constant $k>0$, then $\lambda$ has the lower bound $\lambda \geq n k$. On other hand, if Ric $\geq(n-1) k$ for some constant $k \geq 0$, then $\lambda$ satisfies the lower bound inequality $\lambda \geq(n-1) k / 4+\pi^{2} / \mathrm{D}^{2}(M)$.

Next, we will consider the case of a positive eigenvalue $\lambda$ of $\Delta_{B}$ such that its eigentensor $\varphi$ is a traceless bilinear form. In other words, $\varphi \in C^{\infty}\left(S_{0}^{2} M\right)$, where $S_{0}^{2} M$ is the vector bundle of traceless symmetric bilinear forms. Then, using (4), we have the integral equality

$$
\lambda\langle\varphi, \varphi\rangle=\langle B \varphi, \varphi\rangle+\langle\nabla \varphi, \nabla \varphi\rangle .
$$

By direct computations we obtain the identity

$$
g(B \varphi, \varphi)=(1 / 2) g(K \varphi, \varphi),
$$

where

$$
K:=\operatorname{Ric} \circ \varphi+\varphi \circ \operatorname{Ric}-2 \stackrel{\circ}{R} \varphi
$$

is the Weitzenböck curvature operator of the Lichnerowicz Laplacian (see ([7], p. 54); ([11], p. 388))

$$
\Delta_{L}=\bar{\Delta}+K \text { id }
$$

In addition, we obtain equalities $K(g)=0$ and trace ${ }_{g} K(\varphi)=0$.

Let $\left\{e_{i}\right\}$ be an orthonormal basis of the tangent space $T_{x} M$ at an arbitrary point $x \in M$ such as $\varphi_{x}\left(e_{i}, e_{j}\right)=\lambda_{i}(x) \delta_{i j}$, where $\delta_{i j}$ is the Kronecker symbol and $\sec \left(e_{i} \wedge e_{j}\right)$ is the sectional curvature of the plane $\pi(x)=\operatorname{span}\left\{e_{i}, e_{j}\right\}$, then (see ([11], p. 388))

$$
g(K(\varphi), \varphi)=\sum_{i \neq j} \sec \left(e_{i} \wedge e_{j}\right)\left(\varphi_{i i}-\varphi_{j j}\right) .
$$


Now, let $\Delta_{B}$ be the Bourguignon Laplacian acting on the vector space of $C^{\infty}$-sections of $S_{0}^{2} M$. If we assume $\sec \left(\sigma_{x}\right) \geq K_{\min }>0$ in all directions $\sigma_{x}$ at each point $x \in M$, then from (8) we obtain the integral inequality

$$
\lambda\langle\varphi, \varphi\rangle \geq \frac{1}{2} K_{\min } \int_{M} \sum_{i \neq j}\left(\varphi_{i i}-\varphi_{j j}\right)^{2} \mathrm{~d}_{\operatorname{vol}_{g}}+\langle\nabla \varphi, \nabla \varphi\rangle \geq 0,
$$

for an arbitrary positive eigenvalue $\lambda$ corresponding to a nonzero eigentensor $\varphi \in C^{\infty}\left(S_{0}^{2}\right)$ of $\Delta_{B}$. If the condition trace $g=\sum_{i} \varphi_{i i}=0$ holds, then

$$
\|\varphi\|^{2}=\varphi_{11}^{2}+\varphi_{22}^{2}+\ldots+\varphi_{n n}^{2}=\frac{1}{n} \sum_{i<j}\left(\varphi_{i i}-\varphi_{j j}\right)^{2}
$$

as it equals to the following:

$$
\sum_{i} \varphi_{i i}^{2}=-2 \sum_{i<j} \varphi_{i i} \varphi_{j j}
$$

that is $\left(\sum_{i} \varphi_{i i}\right)^{2}=0$. In this case, from (10) we obtain the integral inequality

$$
\left(\lambda-n K_{\min }\right) \int_{M}\|\varphi\|^{2} \mathrm{dvol}_{g} \geq 0 .
$$

From (11) we conclude that $\lambda \geq n K_{\min }$ for any positive eigenvalue $\lambda$. If the first positive eigenvalue $\lambda=n K_{\min }$, then its corresponding traceless bilinear form $\varphi$ is parallel. In this case, if the holonomy of $(M, g)$ is irreducible, then $\varphi=\mu \cdot g$ for some constant $\mu$. However, in our case, the identity $\operatorname{trace}_{g} \varphi=0$ holds and, consequently, we have $\mu=0$. Thus, the following holds.

Proposition 2. Let the Bourguignon Laplacian $\Delta_{B}$ act on traceless symmetric bilinear forms on a closed Riemannian manifold $\left(M^{n}, g\right)(n \geq 2)$. Then the first positive eigenvalue of $\Delta_{B}$ satisfies the lower bound $\lambda \geq$ $n K_{\min }$ for the minimum $K_{\min }$ of the strictly positive sectional curvature of $(M, g)$. Moreover, if $\lambda=n K_{\min }$, then the traceless symmetric bilinear form $\varphi$ corresponding to $\lambda$ is parallel. In particular, if the holonomy of $(M, g)$ is irreducible, then $\varphi \equiv 0$.

For example, if $(M, g)$ is the standard sphere $\left(S^{n}, g_{0}\right)$, then $\sec (X \wedge Y)=1$ for orthonormal vector fields, $X$ and $Y$. In this case, the first positive eigenvalue $\lambda \geq n$. We can formulate the following corollary.

Corollary 1. Let the Bourguignon Laplacian $\Delta_{B}$ act on traceless symmetric bilinear forms on the standard sphere $\left(S^{n}, g_{0}\right)$ with $n \geq 2$. Then the first positive eigenvalue of $\Delta_{B}$ satisfies the lower bound inequality $\lambda \geq n$.

In the case of the sphere $\left(S^{n}, g_{0}\right)$ we have

$$
B \varphi:=\varphi \circ \operatorname{Ric}-\stackrel{\circ}{R} \varphi=n \varphi, \quad K \varphi=2 n \varphi
$$

for any symmetric bilinear form $\varphi \in C^{\infty}\left(S_{0}^{2} M\right)$. Then we obtain the equality $\Delta_{B} \varphi=(\mu-n) \varphi$ for an arbitrary positive eigenvalue $\mu$ of $\Delta_{L}$ and for some $\varphi \in C^{\infty}\left(S_{0}^{2} M\right)$ corresponding to $\mu$. This means that the eigenvalue $\lambda$ of $\Delta_{B}$, which corresponds to the same bilinear form $\varphi \in C^{\infty}\left(S_{0}^{2} M\right)$, is equal to $\lambda=\mu-n$. The converse is also true.

Consider the Lichnerowicz Laplacian $\Delta_{L}$ acting on traceless and divergence-free symmetric bilinear forms or, in other words, TT-tensors defined on the standard sphere $\left(S^{n}, g_{0}\right)$. In this case, the eigenvalues of $\Delta_{L}$ are given by the formula $\mu_{a}=a(n-1+a)+2(n-1)$ for all $a \geq 2$, see [15], i.e.,

$$
\operatorname{spec}\left(\Delta_{L} \mid T T\right)=\{a(n-1+a)+2(n-1) a \geq 2\} .
$$


Then we immediately obtain the spectrum of the $\Delta_{B}$ acting on the TT-tensors defined on the standard sphere $\left(S^{n}, g_{0}\right)$ :

$$
\operatorname{spec}\left(\Delta_{B} \mid T T\right)=\{a(n-1+a)+(n-2): a \geq 2\} .
$$

Based on this result, we obtain the following.

Proposition 3. The eigenvalues of $\Delta_{B}$ acting on the TT-tensors defined on the standard sphere $\left(S^{n}, g_{0}\right)$ are given by the formula

$$
\lambda_{a}=a(n-1+a)+(n-2), \quad a \geq 2 .
$$

\section{Vanishing Theorems for Harmonic Symmetric Bilinear Forms}

Formula (1) indicates that we may take a symmetric bilinear form $\varphi \in C^{\infty}\left(S^{2} M\right)$ viewed as one form with values in the cotangent bundle. In this case, $\varphi$ is a Codazzi tensor if and only if $d^{\nabla} \varphi=0$. Therefore, we obtain the following statement (e.g., ([2], p. 350)).

Lemma 2. A symmetric bilinear form $\varphi \in C^{\infty}\left(S^{2} M\right)$ on a Riemannian manifold $(M, g)$ is a Codazzi tensor if and only if it is a closed one-form viewed as a one form with values in the cotangent bundle $T^{*} M$.

It was proven in ([5], p. 271) that

$$
\delta^{\nabla} \varphi=-d\left(\operatorname{trace}_{g} \varphi\right)
$$

for an arbitrary Codazzi tensor $\varphi \in C^{\infty}\left(S^{2} M\right)$. At the same time, he defined a harmonic symmetric bilinear form in ([5], p. 270) (e.g., ([2], p. 350)).

Definition 1. A symmetric bilinear form $\varphi \in C^{\infty}\left(S^{2} M\right)$ on a Riemannian manifold $(M, g)$ is harmonic if $\varphi \in \operatorname{Ker} d^{\nabla} \cap \operatorname{Ker} \delta^{\nabla}$.

Based on Definition 1, Lemma 2 and (12), we obtain the following.

Proposition 4. A symmetric bilinear form $\varphi \in C^{\infty}\left(S^{2} M\right)$ on a Riemannian manifold $(M, g)$ is harmonic if and only if it is a Codazzi tensor with constant trace.

Remark 2. Simple examples of bilinear symmetric harmonic forms are the second fundamental form of a hypersurface with constant mean curvature of a Riemannian manifold of constant sectional curvature and the Ricci tensor of a locally conformal flat Riemannian manifold of constant scalar curvature.

Based on (2) and (3), we conclude that the kernel of $\Delta_{B}$ on a closed Riemannian manifold $(M, g)$ is finite-dimensional and satisfies the condition

$$
\operatorname{Ker} \Delta_{B}=\operatorname{Ker} d^{\nabla} \cap \operatorname{Ker} \delta^{\nabla}
$$

Hence, $\Delta_{B}$-harmonic bilinear forms on a closed Riemannian manifold are harmonic symmetric bilinear forms (see ([7], p. 436)). Thus, we have the following.

Proposition 5. Let $\left(M^{n}, g\right)$ be a closed Riemannian manifold. Then, the kernel of the Laplacian $\Delta_{B}$ : $C^{\infty}\left(S^{2} M\right) \rightarrow C^{\infty}\left(S^{2} M\right)$ is a finite dimensional vector space of harmonic symmetric bilinear forms, or, in other words, Codazzi tensors with constant trace. 
It was proven in ([5], p. 281), that a closed orientable four-dimensional Riemannian manifold admitting a non-trivial Codazzi tensor with constant trace must have signature zero (see the definition in ([7], p. 161)). Thus, the following holds.

Proposition 6. Let $\left(M^{4}, g\right)$ be a closed orientable Riemannian manifold. If the kernel of $\Delta_{B}$ is non-trivial, then $(M, g)$ has zero signature.

Using (4), one obtains the Bochner-Weitzenböck formula

$$
\begin{aligned}
(1 / 2) \Delta\left(\|\varphi\|^{2}\right) & =-g(\bar{\Delta} \varphi, \varphi)+\|\nabla \varphi\|^{2} \\
& =-g\left(\Delta_{B} \varphi, \varphi\right)+(1 / 2) g(K \varphi, \varphi)+\|\nabla \varphi\|^{2}
\end{aligned}
$$

for any $\varphi \in C^{\infty}\left(S^{2} M\right)$. Let $\varphi \in C^{\infty}\left(S^{2} M\right)$ be harmonic then (13) can be rewritten as (e.g., (9)):

$$
\Delta\|\varphi\|^{2}=\sum_{i \neq j} \sec \left(e_{i} \wedge e_{j}\right)\left(\varphi_{i i}-\varphi_{j j}\right)+2\|\nabla \varphi\|^{2} .
$$

Recall that a Codazzi tensor $\varphi$ commutes with the Ricci tensor Ric of $(M, g)$ (see )[7], p. 439)); therefore, the eigenvectors of $\varphi$ determine the principal directions of Ric at each point $x \in M$. The converse is also true.

Using (14) and the Hopf maximum principle, we obtain the following.

Lemma 3. Let $(M, g)$ be an open Riemannian manifold and $\varphi$ be a harmonic symmetric bilinear form defined on $M$. If the sectional curvature $\sec \left(e_{i} \wedge e_{j}\right) \geq 0$ for all vectors of the orthonormal basis $\left\{e_{i}\right\}$ of $T_{x} M$, which is determined by the principal directions of the Ricci tensor at an arbitrary point $x \in M$, and $\|\varphi\|^{2}$ has a local maximum in $M$, then $\varphi$ is parallel in $M$. Moreover, if $\sec \left(e_{i} \wedge e_{j}\right)>0$ at a point $x \in M$, then $\varphi$ is trivial at $x$.

Proof. Suppose that $\sec \left(e_{i} \wedge e_{j}\right) \geq 0$ in $M$ then $g(K \varphi, \varphi) \geq 0$. Moreover, if there is a nonzero Codazzi tensor $\varphi$ given in $M$ then from (14) we conclude that $\Delta\|\varphi\|^{2} \geq 0$, i.e., $\|\varphi\|^{2}$ is a nonnegative subharmonic function in $M$. Suppose that $\|\varphi\|^{2}$ has a local maximum at some point $x \in M$, then $\|\varphi\|^{2}$ is a constant function in $M$ according to the Hopf's maximum principle (e.g., [1]). In this case,

$$
\|\nabla \varphi\|^{2}=0 .
$$

In particular, the last equation means that the form $\varphi$ is parallel.

Let $\|\varphi\|^{2}=C$ for some constant $C$, then from (14) we find

$$
g(K \varphi, \varphi)+2\|\nabla \varphi\|^{2}=0 .
$$

Since $\sec \left(e_{i} \wedge e_{j}\right) \geq 0$, this means that

$$
g(K \varphi, \varphi)=0, \quad \nabla \varphi=0 .
$$

If there is a point $x \in M$ such that $\sec \left(e_{i} \wedge e_{j}\right)>0$ then from (14) we find $\varphi_{11}(x)=\ldots=\varphi_{n n}(x)=\lambda$, which is equivalent to $\varphi=(\lambda / n) g$, see ([7], p. 436).

If $(M, g)$ is a closed manifold and a harmonic symmetric bilinear form $\varphi$ is given in a global way on $(M, g)$ then due to the "Bochner maximum principle" for closed manifold it follows the classical Berger-Ebin theorem (see ([7], p. 436) and ([11], p. 388)), which is a corollary of Lemma 3.

Corollary 2. Every harmonic symmetric bilinear form $\varphi \in C^{\infty}\left(S^{2} M\right)$ on a closed Riemannian manifold $(M, g)$ with nonnegative sectional curvature is parallel. Moreover, if $\sec >0$ at some point, then $\varphi$ is trivial. 
Remark 3. It is well known that every parallel symmetric tensor field $\varphi \in C^{\infty}\left(S^{2} M\right)$ on a connected locally irreducible Riemannian manifold $(M, g)$ is proportional to $g$, i.e., $\varphi=\lambda g$ for some constant $\lambda$. Using this statement, we can reformulate Corollary 2 in the following form: Every harmonic bilinear form $\varphi \in C^{\infty}\left(S^{2} M\right)$ on a connected closed and locally irreducible Riemannian manifold $(M, g)$ with nonnegative sectional curvature is trivial.

For example, let $(M, g)$ be a Riemannian symmetric space of compact type, that is a closed Riemannian manifold with non-negative sectional curvature with parallel curvature tensor and positive-definite Ricci tensor (see ([7], p. 196); ([2], pp. 386, 392)). Therefore, we have the following.

Corollary 3. Every harmonic symmetric bilinear form on a Riemannian symmetric manifold of compact type is parallel. If, in addition, the manifold is locally irreducible, then its harmonic symmetric bilinear forms are trivial.

The following theorem supplements the classical Berger-Ebin theorem (see ([11], p. 388) and ([7], p. 436)) for the case of a complete noncompact manifold.

Proposition 7. Let $(M, g)$ be a complete Riemannian manifold with nonnegative sectional curvature. Then there is no a nonzero harmonic form $\varphi \in C^{\infty}\left(S^{2} M\right)$ such that $\int_{M}\|\varphi\| \mathrm{dvol}_{g}<\infty$.

Proof. Let $\varphi \in C^{\infty}\left(S^{2} M\right)$ be a globally defined nonzero harmonic symmetric bilinear form, then $g(K \varphi, \varphi) \geq 0$. Therefore, from (14) we obtain the inequality

$$
\|\varphi\| \Delta(\|\varphi\|)=-(1 / 2) g(K \varphi, \varphi)+\|\nabla \varphi\|^{2}-\|\nabla\| \varphi\|\|^{2} \geq 0,
$$

where $\|\nabla \varphi\|^{2} \geq\|\nabla\| \varphi\|\|^{2}$ by the Kato inequality (see [16]). We conclude that $\|\varphi\|$ is a non-negative subharmonic function on a complete simply connected noncompact Riemannian manifold with nonnegative sectional curvature. In this case, if $\|\varphi\|$ is not identically zero, then $\int_{M}\|\varphi\| \mathrm{d} \operatorname{vol}_{g}=\infty$ (see [17]).

If $(M, g)$ is a hypersurface of an $(n+1)$-dimensional Riemannian manifold of constant sectional curvature then its second fundamental form is a Codazzi tensor (see ([7], p. 436)). Moreover, if $(M, g)$ has constant mean curvature then its second fundamental form is a harmonic bilinear form by Proposition 4. Based on above and Proposition 7, we obtain the following.

Corollary 4. Let $(M, g)$ be a complete simply connected hypersurface with constant mean curvature of an $(n+1)$-dimensional Riemannian manifold of positive constant sectional curvature. If its sectional curvature is nonnegative and the second fundamental form $\varphi$ satisfies the inequality $\int_{M}\|\varphi\| d v_{g}<\infty$, then $(M, g)$ is a spherical space form.

Remark 4. Corollary 4 completes the following result from [11]: If $(M, g)$ is a compact minimal hypersurface of a Euclidean sphere and $(M, g)$ has strictly positive sectional curvature, then $(M, g)$ is an equator of the sphere.

Author Contributions: All authors contributed equally and significantly in writing this article. All authors have read and agreed to the published version of the manuscript.

Funding: This research received no external funding.

Conflicts of Interest: The authors declare no conflict of interest.

\section{References}

1. Agricola, I.; Friedrich, T. Global Analysis. Differential Forms in Analysis, Geometry and Physics; AMS: Providence, RI, USA, 2002.

2. Petersen, P. Riemannian Geometry; Springer: Basel, Switzerland, 2016. 
3. Stepanov, S.E.; Tsyganok, I.I. Conformal Killing $L^{2}$-forms on complete Riemannian manifolds with nonpositive curvature operator. J. Math. Anal. Appl. 2018, 458, 1-8. [CrossRef]

4. Catino, G.; Mantegazza, C.; Mazzieri, L. A note on Codazzi tensors. Math. Ann. 2015, 362, $629-638$. [CrossRef]

5. Bourguignon, J.-P. Les variétés de dimension 4 á signature non nulle dont la courbure est harmonique sont d'Einstein. Invent. Math. 1981, 63, 263-286. [CrossRef]

6. Lablée, O. Spectral Theory in Riemannian Geometry; EMS Textbooks in Mathematics; EMS: Zúrich, Switzerland, 2015.

7. Besse, A. Einstein Manifolds; Springer: Berlin/Heidelberg, Germany, 1987.

8. Hasanis, T.; Vlachos, T. Hypersurfaces and Codazzi tensors. Monat. Math. 2008, 154, 51-58. [CrossRef]

9. Shandra, I.G.; Stepanov, S.E.; Mikeš, J. On higher order Codazzi tensors on complete Riemannian manifolds. J. Ann. Glob. Anal. Geom. 2019, 56, 429-442. [CrossRef]

10. Absan, Z.; Ali, M. Curvature tensor for space-times of General Relativity. Int. J. Geom. Methods Mod. Phys. 2017, 14, 1750078.

11. Berger, M.; Ebin, D. Some decomposition of the space of symmetric tensors on a Riemannian manifold. J. Diff. Geom. 1969, 3, 379-392. [CrossRef]

12. Bourguignon, J.-P. Formules de Weitzenbök en dimension 4. In Seminaire A. Besse sur la géometrie Riemannienne Dimension 4; Cedic. Ferman: Paris, France, 1981; pp. 156-177.

13. Craioveanu, M.; Puta, M.; Rassias, T.M. Old and New Aspects in Spectral Geometry; Kluwer Academic Publishers: Dordrecht, The Netherlands, 2013.

14. Yang, D. Lower bound estimates of the first eigenvalue for compact manifolds with positive Ricci curvature. Pac. J. Math. 1999, 190, 383-398. [CrossRef]

15. Boucetta, M. Spectrum of the Lichnerowicz Laplacians on the spheres and the real projective spaces. Publ. Mater. 1999, 43, 2451-2483.

16. Calderbank, D.M.J. Refined Kato inequalities and conformal weights in Riemannian geometry. J. Funct. Anal. 2000, 173, 214-255. [CrossRef]

17. Greene, R.E.; Wu, H. Integrals of subharmonic functions on manifolds of nonnegative curvatures. Invent. Math. 1974, 27, 265-298. [CrossRef]

(C) 2020 by the authors. Licensee MDPI, Basel, Switzerland. This article is an open access article distributed under the terms and conditions of the Creative Commons Attribution (CC BY) license (http://creativecommons.org/licenses/by/4.0/). 